\title{
Dental Alloy Sorting By the Thermoelectric Method
}

\author{
Masafumi Kikuchi
}

\section{ABSTRACT}

Objectives: A nondestructive, rapid, and practical method of dental alloy sorting is desirable. In this study, the hypothesis to be tested is that dental alloys show significantly different and high thermoelectric power values, on the basis of which alloy sorting is possible.

Methods: Six silver-colored commercial dental casting alloys are used in this study: two silver alloys, one gold-silver-palladium alloy, one cobalt-chromium alloy, one nickel-chromium alloy, and one titanium alloy. The thermoelectric power of their castings was determined against constantan using a simple apparatus developed in a previous study. Linear least square fitting was applied to the measured thermal-EMF-temperature curve to determine the thermoelectric power for the temperature ranges of 298-303 K (temperature difference $\Delta t=5 \mathrm{~K}), 298-308 \mathrm{~K}(\Delta \mathrm{t}=10 \mathrm{~K}), 298-313 \mathrm{~K}(\Delta \mathrm{t}=15 \mathrm{~K})$, and 298-318 $\mathrm{K}(\Delta \mathrm{t}=20 \mathrm{~K})$. The results were analyzed using one-way ANOVA and by the Scheffé's test at a significance level of $\alpha=0.01$.

Results: When the temperature difference was $10 \mathrm{~K}$ or less, the difference in the thermoelectric powers of the alloys was not always statistically significant. However, when the temperature difference was $15 \mathrm{~K}$ or more, the thermoelectric powers of the six alloys differed significantly.

Conclusions: The results indicated the feasibility of rapid sorting of specific dental alloys by the thermoelectric method, provided a sufficiently large temperature difference is achieved. (Eur J Dent 2010;4:66-70)

Key words: Dental casting alloy; Alloy sorting; Thermoelectric power; Thermal electromotive force; Thermocouple.

\section{INTRODUCTION}

Various alloys such as gold alloys, silver alloys, and cobalt-chromium alloys are used for dental applications. ${ }^{1}$ Alloys with similar appearance can-

- a Division of Dental Biomaterials, Tohoku University Graduate School of Dentistry, Sendai, Miyagi 980-8575, Japan.

- Corresponding author: Masafumi Kikuchi, Division of Dental Biomaterials, Tohoku University Graduate School of Dentistry, 4-1 Seiryo-machi, Aoba-ku, Sendai, Miyagi 980-8575, Japan.

Phone: +81-22-717-8317

Fax: +81-22-717-8319

E-mail: kikuchi@mail.tains.tohoku.ac.jp not always be distinguished once they are cast or machined, and the alloys lose their original form or markings. Alloy sorting (identification) of prostheses made of unknown alloys is necessary when additional prostheses are required over the existing ones; the chemical composition of the new prosthesis must match that of the existing one to minimize the risk of galvanic corrosion. Alloy sorting is also important to identify metallic allergies. Unwanted mixing of alloys can lead to problems if the alloys are reused in dental laboratories.

The most reliable method for identifying a solid alloy is to carry out elemental analysis using so- 
phisticated and rather expensive instruments such as an X-ray photoelectron spectrometer and an electron probe microanalyzer. These instruments cannot be used intraorally; thus, to analyze the prosthesis alloy, a certain amount of sample must be removed from it. Therefore, a portable device that uses a non-destructive and rapid identification technique is desirable. Such devices are called metal testers or alloy sorters; they are commercially available and are used in industrial applications. ${ }^{2,3}$ These devices typically use thermoelectric methods to measure the thermal electromotive force (EMF).

When two different metals are connected in a loop, the thermal EMF is determined from the thermoelectric power (measured in units of $\mathrm{V} \mathrm{K}^{-1}$ and refers to the voltage difference per unit increment in temperaturel of the metals and the temperature difference between the two junctions. If the thermoelectric power and the temperature of one of the two junctions are known, the thermal EMF can be used to determine the other junction temperature. This principle is the same as that of temperature measurements using a thermocouple. ${ }^{4}$ If the temperature difference is known, the thermal EMF can be used to determine the thermoelectric power of a combination of two metals. It should be noted that the thermal EMF is not affected by the shape or size of the metals. The determination of thermoelectric power can be applied to alloy sorting; for this, it is important to develop a table that includes the thermoelectric powers of various dental alloys measured against a standard metal. The measurement of the thermal EMF requires a simple direct-current circuit. The limitation of this approach is that alloys with the same thermoelectric power need not necessarily have the same chemical compositions. However, when the number of different types of alloys tested is limited and their thermoelectric powers differ signifi- cantly, alloy sorting by the thermal EMF measurement can be practically feasible. Another concern about this method is its intraoral application when the thermoelectric power of the alloy is low; intraoral application in this case is difficult because it is necessary to achieve a high temperature difference between the junctions to obtain sufficient voltage against the background noise.

Thus far, a method to predict the thermoelectric power of an alloy on the basis of its chemical composition has not been proposed. On searching various literatures, I found no reports on the thermoelectric properties of dental alloys. The hypothesis to be tested in the present study is that the dental alloys exhibit significantly different and high thermoelectric power values, and this property can be used in alloy sorting. The thermoelectric powers of six silver-colored commercial dental-casting alloys were determined to verify the feasibility of dental alloy sorting by thermoelectric method.

\section{MATERIALS AND METHODS}

Preparation of specimens

The six dental-casting alloys used in this study include two silver alloys (Miro 3 (M3) and Casting Silver S (CS), GC, Tokyo, Japan), one gold-silverpalladium alloy (Castwell M.C. 12\% Gold (CW), $\mathrm{GC}$ ), one cobalt-chromium alloy (Cobaltan (CO), Shofu, Kyoto, Japan), one nickel-chromium alloy (Dent-Nickel (DN), Shofu), and one titanium alloy (T-Alloy Tough (TA), GC); these alloys are summarized in Table 1. To prepare the specimens of these alloys (diameter: $2 \mathrm{~mm}$, length: $30 \mathrm{~mm}$ ), a rod pattern (diameter: $2 \mathrm{~mm}$ ) was invested. A cristobalite investment (Cristoquick 20, GC) was used for the low casting-temperature alloys (M3, CS, and CW). A phosphate-bonded investment (Ceravest G, GC) was used for $\mathrm{CO}$ and DN. A magnesia investment (Selevest CB, Selec, Osaka, Japan) was used for

Table 1. Dental casting alloys used in this study and their chemical compositions (catalog value).

\begin{tabular}{l|c|c|c}
\hline Alloy (Specification) & Product name & Abbr. & Main components, mass\% \\
\hline Silver alloy (JIS T6108 Type 1) & Miro 3, GC & M3 & Ag: 77\%, Sn: 18\%, Zn: 5\% \\
Silver alloy (JIS T6108 Type 2) & Casting Silver S, GC & CS & Ag: 70.5\%, In: 24\%, Zn: 2.5\%, Pd: 1\% \\
Gold-Silver-Palladium alloy (JIS T6106 Type 2) & Castwell M.C., GC & CW & Ag: 46\%, Pd: 20\%, Cu: 20\%, Au: 12\% \\
Cobalt-Chromium alloy (JIS T6115) & Cobaltan, Shofu & CO & Co: 63\%, Cr: 29\%, Mo: 6\% \\
Nickel-Chromium alloy (NA) & Dent-Nickel, Shofu & DN & Ni: 63\%, Cr: 15\%, Nb: 5\%, Mn: 5\% \\
Ti-6Al-7Nb (ASTM F1295) & T-Alloy Tough, GC & TA & Ti: 86.5\%, Al: 6\%, Nb: 7\% \\
\hline
\end{tabular}


TA. The molds for the specimens were burnt out according to the investment and alloy manufacturers' instructions. The alloys with low casting temperatures were cast into the molds using the dental casting unit Caspak C602 (Dentronics, Tokyo, Japan). The dental casting unit ArgoncasterC (Shofu) was used for CO and DN, while a titanium casting unit (Ticast Super R, Selec, Osaka, Japan) was used for TA. After casting, the molds were bench cooled. Three specimens were prepared for each alloy.

\section{Determination of thermoelectric power}

The thermal EMF of the specimens was measured using the apparatus shown in Figure 1; this apparatus was developed in a previous study to determine the thermoelectric power against constantan. ${ }^{5}$ The validity of this apparatus is discussed in the concerned paper. A constantan wire (diameter: 1.0 mm, JIS type-T, Hayashidenko, Tokyo, Japan) was pressed against the specimen under approximately $3 \mathrm{~N}$ using a spring. A thin copper wire IVFF $1.25 \mathrm{~mm}^{2}$, single element wire was used at a time, diameter: $0.18 \mathrm{~mm}, \mathrm{KHD}$, Osaka, Japan) was inserted between the specimen and the constantan wire. Two temperature sensors (LM35CAZ, National Semiconductor, Santa Clara, CA, USAl were installed near the cold junctions. The specimen-constantan thermal EMF and the copper-constantan thermal EMF were obtained using a computer with an analog-to-digital interface (DAQCard-6036E, National Instruments, Austin, TX, USA). The hot junction was heated starting from room temperature, which was below $298 \mathrm{~K}$, by heating the end of the thin copper wire using a gas torch (Handy Torch Ortho, YDM, Tokyo, Japan). The temperature of the hot junction was estimated from the copper-constantan thermal EMF and the average temperature of the two temperature sensors using the formulas for the type-T thermocouple. ${ }^{6}$ The electric contact areas of the specimens and constantan wire were previously ground with 1000-grit SiC abrasive papers. A new thin copper wire was used for every measurement. The measurement was performed three times on each specimen.

A linear least-square fitting was performed on the measured thermal-EMF-temperature curve to determine the thermoelectric power of each specimen against constantan at temperatures ranges of $298-303 \mathrm{~K}$ (temperature difference $\Delta t=5$ $\mathrm{K}), 298-308 \mathrm{~K}(\Delta \mathrm{t}=10 \mathrm{~K}), 298-313 \mathrm{~K}(\Delta \mathrm{t}=15 \mathrm{~K})$, and $298-318 \mathrm{~K}(\Delta \mathrm{t}=20 \mathrm{~K})$. The goodness of the fit was examined by calculating the coefficient of determination $\left(R^{2}\right)$. To test the statistical distinctness of the thermoelectric power of each alloy, the results were analyzed by one-way ANOVA and the Scheffé's test at a significance level of $\alpha=0.01$.

\section{RESULTS}

In the case of each alloy, when the temperature difference was $5 \mathrm{~K}$, the mean $\mathrm{R}^{2}$ value was greater than 0.98 . When the temperature difference was $10 \mathrm{~K}$ or more, the mean $\mathrm{R}^{2}$ value was greater than 0.99 . The thermoelectric power values of the six dental alloys against constantan in the four temperature ranges are summarized in Figure 2. When the temperature difference is $10 \mathrm{~K}$ or less, the difference between the thermoelectric powers of the alloys was not always statistically significant (Figure 2 (a), (b)). As the temperature difference increased, the standard deviation decreased. When the temperature difference was $15 \mathrm{~K}$ and more, the thermoelectric power values of the alloys were significantly different $(P<.01)$ (Figure 2 (c), (d)). The thermoelectric power value of each alloy increased slightly with an increase in the temperature difference.

\section{DISCUSSION}

The results suggest that dental alloy sorting by thermoelectric method is feasible when the number of alloys being sorted is limited. At the same time, the results revealed certain limitations of this method. When the hot junction temperature was relatively low and the temperature difference was small, the difference in the thermoelectric power values of some alloys was not significant. Dental alloy sorting by this method may become difficult when the difference in the thermoelectric power values of the various alloys is very small or the dispersion of the measured values is large.

$\mathrm{R}^{2}$ values close to 1 indicated that the linear regression fit of the experimental data was good. However, a slight increase in the thermoelectric power value was observed with increasing temperature range. This result was due to the nonlinearity in the relationship between temperature and thermal EMF, which is a typical feature of thermocouples. ${ }^{4}$ The thermal EMF is a function of 
temperature, and within the measured temperature range, the measured function was slightly

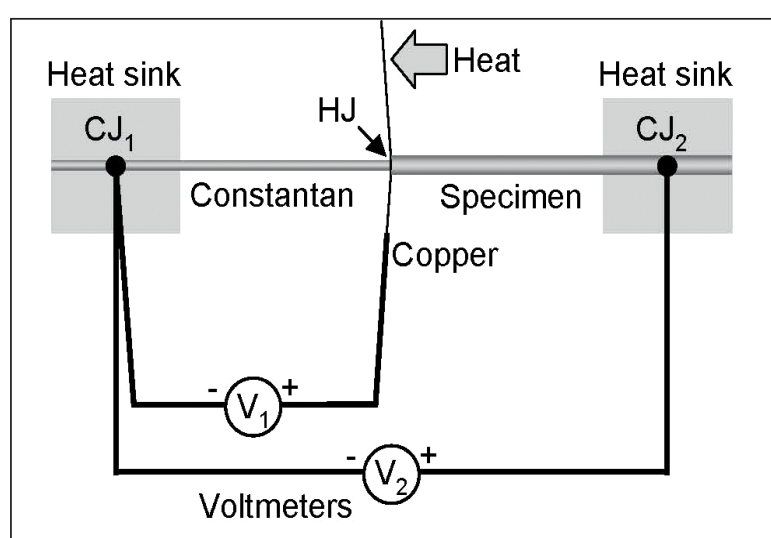

Figure 1. Schematic diagram of apparatus used for measuring thermal EMF of dental alloys against constantan and cold junctions $\left(\mathrm{CJ}_{1}\right.$ and $\left.\mathrm{CJ}_{2}\right)$ at ambient temperature. $\mathrm{V}_{1}$ is the thermal EMF of copper against constantan and is used to measure the hot-junction $(\mathrm{HJ})$ temperature. $V_{2}$ is the thermal EMF of the specimen against constantan. convex and increased monotonically. Therefore, an appropriate temperature range should be selected for the measurement of thermoelectric power.

It is known that in an oral cavity, when two dissimilar alloys are in electrical contact, a galvanic couple is produced, and a corrosive galvanic current flows between the alloys. ${ }^{7}$ It may be worth mentioning that when two dissimilar alloys are electrically contacted and a thermocouple is formed, a weak thermoelectric current could also flow between the alloys; this thermoelectric current will depend on the difference between the thermoelectric power of the two alloys and the thermal gradient in the oral cavity.

To realize dental alloy sorting on the basis of thermoelectric power, two methods are evidently feasible. One is the determination of the ther-

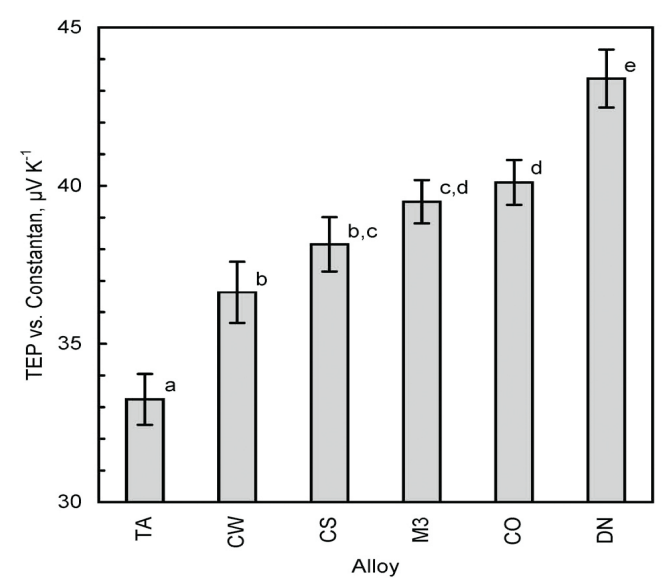

(a) $298-303 \mathrm{~K}(\Delta \mathrm{t}=5 \mathrm{~K})$

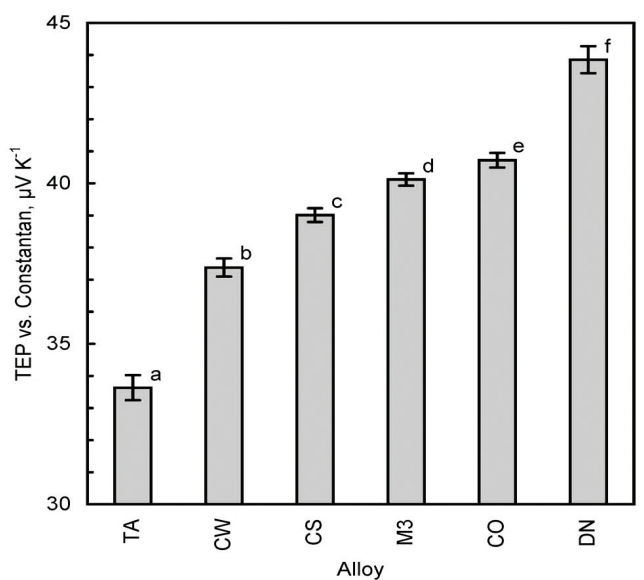

(c) $298-313 \mathrm{~K}(\Delta \mathrm{t}=15 \mathrm{~K})$

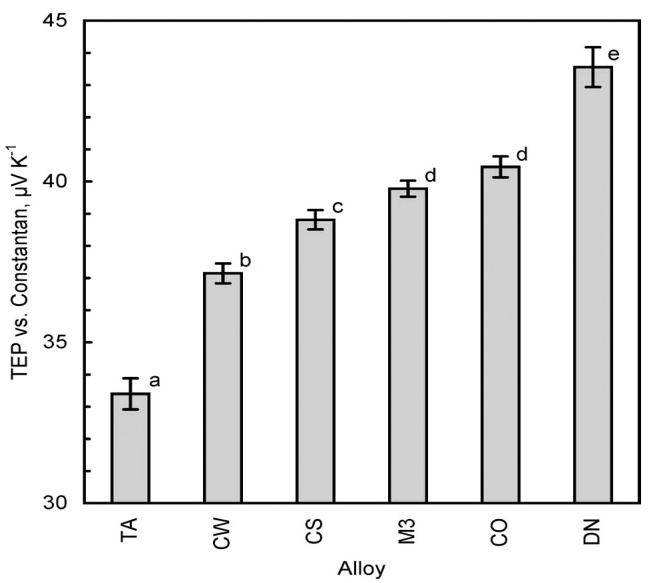

(b) $298-308 \mathrm{~K}(\Delta t=10 \mathrm{~K})$

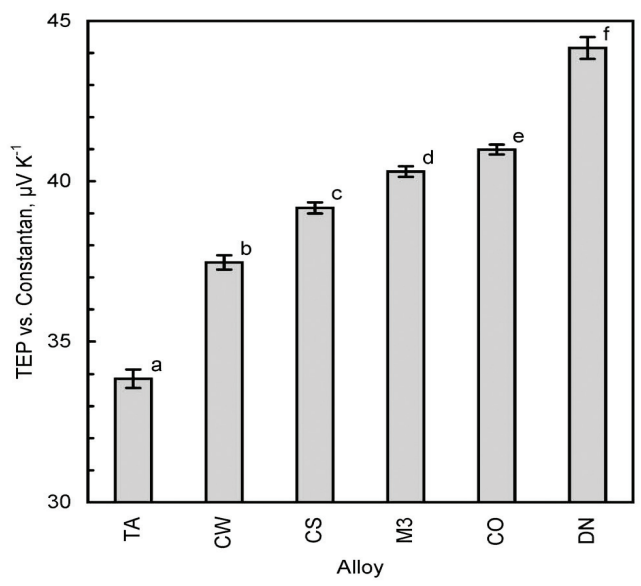

(d) $298-318 \mathrm{~K}(\Delta \mathrm{t}=20 \mathrm{~K})$

Figure 2. Thermoelectric power values of dental alloys. The error bar represents the standard deviation $(\mathrm{n}=9)$. Identical letters indicate that the values are not statistically different $(P>$.01). (a) 298-303 K ( $\Delta \mathrm{t}=5 \mathrm{~K})$; (b) 298-308 K ( $\Delta \mathrm{t}=10 \mathrm{~K})$; (c) 298-313 K ( $\Delta \mathrm{t}$ $=15 \mathrm{~K}) ;(\mathrm{d}) 298-318 \mathrm{~K}(\Delta \mathrm{t}=20 \mathrm{~K})$. 
moelectric power of an unknown alloy against a standard alloy, as shown in the present study; then, this determined value can be matched with the closest value in a previously created table that lists the thermoelectric power values of dental alloys (direct instrumentation). However, it may not be easy to carry out this method intraorally because it is not always easy to control both the hot- and cold-junction temperatures.

The other method is the use of probes made of various known alloys to find a probe that generates zero thermal EMF, as shown in Figure 3 (comparative instrumentation). Although the number of alloys for which this method can be used is limited by the number of probes available, this method is probably more robust against variations in environmental conditions because it is based on the principle that for two identical alloys, the thermal EMF is zero, irrespective of the junction temperatures, provided the two cold-junction temperatures are the same llaw of homogeneous metals). ${ }^{4} \mathrm{~A}$ high hot-junction temperature implies a high output voltage and thus high reliability of the method. In practice, there is an upper limit on the temperature which can be applied intraorally. The hot-junction temperature can be increased by minimizing the hot-junction area and the heating time, while the bulk temperature of the target alloy remains unchanged. As for the two cold junctions, cold-temperature compensation can be provided automatically. In both the methods, acceptance limits should be adjusted by taking into

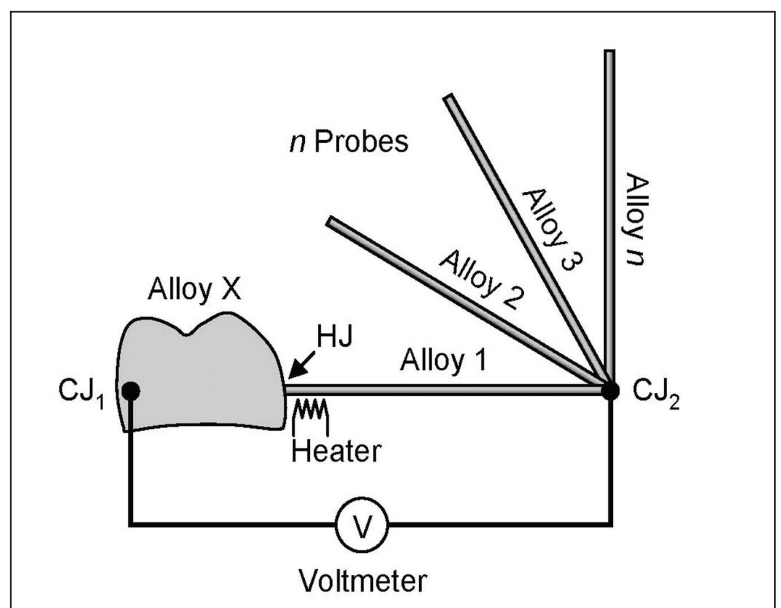

Figure 3. Schematic diagram of dental alloy sorter example. The hot junction (HJ) formed by an unknown alloy (Alloy $\mathrm{X}$ ) and one of the probes made of known alloys (Alloys $1,2,3, \ldots, n$ ) is temporarily heated by the heater. If the temperatures of the two cold junctions ( $\mathrm{CJ}$, and $\mathrm{CJ}_{2}$ ) are the same and the two alloys are identical, the voltmeter will show zero thermal EMF, regardless of the hot-junction temperature. consideration the deviation of the measured value from the standard one. Further study on the thermoelectric properties of a larger variety of dental alloys is required before the use of this method in clinical applications.

\section{CONCLUSIONS}

In this study, the thermoelectric powers of six commercial dental-casting alloys were determined to verify the feasibility of dental alloy sorting by thermoelectric method. Despite certain limitations of the present experiment, the following conclusions are drawn:

- The thermoelectric method can be applied to the rapid sorting of specific dental alloys when a sufficiently large temperature difference is achieved.

- The discrimination of any two alloys is more reliable when the difference between their thermoelectric power values is more.

\section{REFERENCES}

1. O'Brien WJ. Dental materials and their selection. 3rd ed. Carol Stream: Quintessence Pub; 2002.

2. Mix PE. Introduction to nondestructive testing: A training guide. 2nd ed. Hoboken: Wiley; 2005:450-456.

3. ASTM. E977-05 Standard practice for thermoelectric sorting of electrically conductive materials. In: Annual book of ASTM standards 03.03 Nondestructive testing. West Conshohocken: ASTM Int; 2006:423-425.

4. ASTM. Manual on the use of thermocouples in temperature measurement, 3rd ed. Philadelphia: ASTM Int; 1970.

5. Kikuchi M. The use of cutting temperature to evaluate the machinability of titanium alloys. Acta Biomater 2009;5:770775.

6. JIS C1602-1995 Thermocouples. In: JIS HB Heat treatment. Tokyo: Japanese Standards Association; 2002:653-698.

7. Mueller HJ. Tarnish and corrosion of dental alloys. In: ASM Handbook Vol. 13 Corrosion. Materials Park: ASM Int; 2001:1336-1366. 